\title{
Cranial Afferent Glutamate Heterosynaptically Modulates GABA Release onto Second-Order Neurons via Distinctly Segregated Metabotropic Glutamate Receptors
}

\author{
Young-Ho Jin, Timothy W. Bailey, and Michael C. Andresen \\ Department of Physiology and Pharmacology, Oregon Health and Science University, Portland, Oregon 97239-3098
}

\begin{abstract}
The balance between excitation and inhibition dictates central integration. Glutamatergic and GABAergic neurotransmission dominate this process. Cranial primary afferents enter the brainstem to release glutamate (Glu) onto second-order neurons within the caudal nucleus tractus solitarius (NTS) to initiate autonomic reflexes. The simplest pathways for these reflexes contain as few as two central neurons, but display robust frequency-dependent behavior. Within NTS, multiple metabotropic Glu receptors (mGluRs) are present, but their roles are poorly understood. Using synaptically discriminated second-order NTS neurons in brainstem slices and mechanically dissociated NTS neurons with intact boutons, we show that Glu differentially controls GABA release via distinct presynaptic mGluRs. In second-order NTS neurons recorded in slices, activation of primary afferents at frequencies as low as 10 shocks per second released sufficient Glu to alter rates of spontaneous IPSCs (sIPSCs). In both approaches, group I mGluRs increased GABA release in some neurons, but, on different neurons, group II and group III mGluRs decreased the SIPSC rate. mGluR actions were remarkably rapid, with onset and reversal beginning within $100 \mathrm{msec}$. In all cases, mGluR actions were exclusively presynaptic, and mGluRs did not alter postsynaptic properties in second-order neurons in either slices or isolated neurons. Tests with capsaicin and $\alpha \beta$-methylene ATP suggest that myelinated and unmyelinated afferent pathways engage both mGluR-GABA mechanisms. Afferent Glu spillover provides heterosynaptic cross talk with GABAergic inhibition in NTS. This process may critically shape the dynamic character and use dependence for cranial afferent transmission at the first stage of autonomic reflexes.
\end{abstract}

Key words: presynaptic modulation; autonomic; brainstem; nucleus tractus solitarius; GABA; afferent; baroreceptor; sensory neurons; slice; glutamate; metabotropic; heterosynaptic

\section{Introduction}

Metabotropic glutamate receptors (mGluRs) shape both glutamatergic and GABAergic synaptic signaling to complement ionotropic mechanisms at nerve terminals (Conn and Pin, 1997; Cartmell and Schoepp, 2000; Schoepp, 2001). Expression of mGluRs at GABAergic nerve terminals provides central circuits with powerful heterosynaptic interactions. Primary afferents release glutamate (Glu) to initiate central processing of sensory inputs within the spinal dorsal horn (Headley and Grillner, 1990). Visceral organs (e.g., the heart) are often dually innervated with a second set of primary afferent neurons from cranial nerve ganglia, and these cranial afferents directly enter the brainstem to synapse on second-order neurons within the caudal nucleus tractus solitarius (NTS) (Andresen and Kunze, 1994; Andresen et al., 2001). These cranial afferent pathways regulate homeostatic life support systems and are linked to a wide spectrum of neurotransmitters (Pilowsky and Goodchild, 2002). Some of these basic autonomic

Received May 21, 2004; revised Aug. 17, 2004; accepted Aug. 31, 2004.

This work was supported by National Institutes of Health Grants HL-41119 (M.C.A.), HL-56460 (M.C.A.), and HL-70433 (T.W.B.).

Correspondence should be addressed to Dr. Michael C. Andresen, Department of Physiology and Pharmacology, Oregon Health and Science University, Portland, OR 97239-3098. E-mail: andresen@0HSU.edu.

DOI:10.1523/JNEUROSCI.1991-04.2004

Copyright $\odot 2004$ Society for Neuroscience $\quad$ 0270-6474/04/249332-09\$15.00/0 reflex pathways traverse as few as one to two central neurons beyond second-order NTS neurons before exiting the CNS (e.g., the cardiac parasympathetic pathway) (Standish et al., 1995; Wang et al., 2001). Such simple pathway architecture demands very compact integrative mechanisms to account for the substantial frequency dependence and plasticity in autonomic central circuits. The perisomatic concentration of large afferent boutons is an interesting feature of second-order NTS neurons (Mendelowitz et al., 1992; Sekizawa et al., 2003; Doyle et al., 2004). Because GABA terminals are closely adjacent to Glu terminals (Jin et al., 2004), Glu and GABA transmission may intimately interact on second-order NTS neurons.

The mGluR family includes eight single polypeptide chain receptors that signal via coupling to G-proteins (Conn and Pin, 1997). Such mGluRs regulate excitability by presynaptic and postsynaptic mechanisms that increase or decrease transmission depending on neuronal phenotype (Conn and Pin, 1997; Cartmell and Schoepp, 2000; Schoepp, 2001). Immunoreactivity and mRNA evidence suggest that all of the mGluR subunits are found within NTS (Hay et al., 1999; Hoang and Hay, 2001; Pamidimukkala et al., 2002). Solitary tract (ST) activation of cranial afferent axons reliably elicits large EPSCs (Doyle and Andresen, 2001), and many second-order NTS neurons receive longer latency, GABAergic IPSCs synched to ST shocks (Andresen and Yang, 
1990). ST afferent synaptic transmission substantially depresses at moderate frequencies (Andresen and Yang, 1995). Frequencydependent depression in NTS with cranial afferent activation is reduced by mGluR blockade (Liu et al., 1998). Early work in NTS (Feldman and Felder, 1991) suggested complex interactions between afferent activation and GABA with mGluR participation in regulating ST-evoked responses (Glaum et al., 1993). The precise function that mGluRs play within NTS pathways is less clear.

Using a combination of recordings from second-order NTS neurons in brainstem slices together with isolated, mechanically dispersed NTS neurons, we tested whether mGluR activation modulates GABA release onto medial NTS neurons. Surprisingly, the results identify a distinct segregation of group I from group II and group III mGluRs on GABA terminals on different pools of second-order NTS neurons. Furthermore, these distinct distributions provide opposite and rapid regulation of GABA release, which may represent important mechanisms underlying pathway-specific frequency dependence in brainstem circuits.

\section{Materials and Methods}

NTS slices. Hindbrains of male Sprague Dawley rats (Charles River Laboratories, Wilmington, MA) were prepared from 3- to 8-week-old rats for slice recordings (Doyle et al., 2004) and from 2- to 3-week-old rats for dissociated neurons as described previously (Jin et al., 2004). All of the animal procedures were conducted with the approval of the University Animal Care and Use Committee in accordance with the United States Public Health Service Policy on Humane Care and Use of Laboratory Animals and National Institutes of Health Guide for the Care and Use of Laboratory Animals. The hindbrain was removed and placed in ice-cold artificial CSF (ACSF) composed of the following (in mM): $125 \mathrm{NaCl}, 3$ $\mathrm{KCl}, 1.2 \mathrm{KH}_{2} \mathrm{PO}_{4}, 1.2 \mathrm{MgSO}_{4}, 25 \mathrm{NaHCO}_{3}, 10$ dextrose, and $2 \mathrm{CaCl}_{2}$, and bubbled with $95 \% \mathrm{O}_{2}-5 \% \mathrm{CO}_{2}$. The medulla was trimmed to a $1 \mathrm{~cm}$ block (rostral-caudal) centered on the obex. A wedge of tissue was removed from the ventral surface to align the ST within a cutting plane that contained $>1 \mathrm{~mm}$ of ST in the same plane as the NTS (Doyle et al., 2004) when mounted in a vibrating microtome (VT-1000S; Leica, Nussloch, Germany). Slices (250 $\mu \mathrm{m}$ thick for slice recording and 150-170 $\mu \mathrm{m}$ thick for dissociation) were cut with sapphire blades (Delaware Diamond Knives, Wilmington, DE).

Slice recordings. In horizontal brain slice experiments, we tested whether afferent Glu released from ST fibers could activate mGluR receptors on GABA terminals of second-order neurons from the medial subnucleus of subpostremal NTS. For these experiments, the horizontal brainstem slice is optimized for electrophysiological identification of second-order neurons (Doyle et al., 2004). Neurons were visualized within NTS using rapidly refreshed digital images acquired using infrared illumination and differential interference contrast optics (40× water immersion lens) on an Axioskop FS2 microscope (Zeiss, Thornwood, NJ) coupled to an infrared sensitive camera Axiocam (Zeiss). We selected neurons for recording that were located medial $(<500 \mu \mathrm{m})$ and adjacent to the ST. In general, recordings were made rostral to the obex, indicated by the caudal end of the fourth ventricle. Voltage clamp was performed in the ruptured, whole-cell configuration (Axopatch 200A, Axon Instruments, Foster City, CA). Recording electrodes (2.5-3.5 M $\Omega$ ) were filled with an intracellular solution containing the following (in mM): $10 \mathrm{NaCl}$, $40 \mathrm{KCl}, 90 \mathrm{~K}$ gluconate, 11 EGTA, $1 \mathrm{CaCl}_{2}, 2 \mathrm{MgCl}_{2}, 10$ HEPES, 2 $\mathrm{Na}_{2} \mathrm{ATP}$, and $0.2 \mathrm{Na}_{2} \mathrm{GTP}, \mathrm{pH} 7.3$ (295 mOsm). Data were filtered at 3 $\mathrm{kHz}$ and then sampled at $50-100 \mathrm{kHz}$ by pClamp 8 software (Axon Instruments). A concentric bipolar electrode (200 $\mu \mathrm{m}$ diameter; Frederick Haer, Bowdoinham, ME) placed within the stripe of ST delivered stimuli to afferent axons. Because the stimulation electrode was placed 1-3 mm from the site of the recorded neuron soma, we minimized the possibility of direct focal stimulation of non-ST axons [Doyle et al. (2004), their Fig. 1] or local cell bodies (Titz and Keller, 1997). The second-order neurons were identified using jitter $(<150 \mu \mathrm{sec})$, synaptic failure rates, and ionotropic GluR antagonists as described previously (Doyle and Andresen, 2001; Doyle et al., 2004). For the jitter analysis, bursts of five ST stimuli every $3 \mathrm{sec}$ (i.e., $0.3 \mathrm{~Hz}$; shock duration, $0.1 \mathrm{msec}$ ) were generated at burst frequencies of $50 \mathrm{~Hz}$ with a Master- 8 isolated programmable stimulator (AMPI, Jerusalem, Israel). Synaptic latencies were measured as the time between the onset of the stimulus artifact and the onset of the synaptic current. After identification of neurons with second-order synaptic characteristics, application of NBQX (20 $\mu \mathrm{M}$; non-NMDA antagonist) and D-(-)-2-amino-5-phosphonopentanoic acid [AP-5; $100 \mu \mathrm{M}$; NMDA receptor (NMDAR) antagonist] completely blocked ST-evoked synaptic currents as well as spontaneous EPSCs. This condition of complete GluR ionotropic blockade was used to isolate IPSCs but permitted ST stimulation to release afferent Glu while we observed spontaneous GABAergic IPSCs. Identical ST stimulus parameters from the jitter analysis (shock intensity and duration) were used to create trains of stimuli for the afferent Glu release protocols used in slices. The three stimulus train protocols to evoke ST release of Glu were composed of 2, 5, and 10 stimuli delivered in bursts every $0.5 \mathrm{sec}$ and were designated T1, T2, and T3, respectively. The intraburst frequency (shock to shock) was fixed at $50 \mathrm{~Hz}$, but the total number of ST shocks delivered in each burst was increased by extending the burst duration.

Mechanical dissociation. The brainstem slices described above were preincubated $\left(1-3 \mathrm{hr}\right.$ at $\left.31^{\circ} \mathrm{C}\right)$ in well bubbled ACSF before mechanical dispersion. Then the brainstem slices were transferred to $35 \mathrm{~mm}$ Petri dishes (Falcon 1008; Becton Dickinson, Franklin Lakes, NJ) filled with standard external solution containing the following (in $\mathrm{mM}$ ): $150 \mathrm{NaCl}, 5$ $\mathrm{KCl}, 1 \mathrm{MgCl}_{2}, 2 \mathrm{CaCl}_{2}, 10$ HEPES, and 10 glucose ( $\mathrm{pH}$ was adjusted to 7.4 with Tris-base). A glass pipette was pulled to a fine tip and fire-polished to a final tip size of $100-120 \mu \mathrm{m}$ (outer diameter). The polished pipette was mounted in a custom-made vibrator held by a micromanipulator (Jin et al., 2004). Subpostremal portions of NTS medial to the visible ST (identical to the targeted recording region in slices) were identified for dispersion using a stereomicroscope, and the oscillating tip was lowered to the surface within this subregion of the nucleus. The pipette oscillated at $30 \mathrm{~Hz}$ horizontally, with excursions of $100-300 \mu \mathrm{m}$. The pipette tip was moved slowly using the micromanipulator to circumscribe an area of the subnucleus generally from the most caudal end of the fourth ventricle rostrally up to $500 \mu \mathrm{m}$ and medial from the ST to within $50 \mu \mathrm{m}$ of the edge of the fourth ventricle. Neurons were dissociated from the top 100 $\mu \mathrm{m}$ from the dorsal surface of the slices. After the removal of the slice, dispersed neurons were allowed to settle and adhered to the bottom of the dish within $20 \mathrm{~min}$.

For voltage-clamp recording in dissociated cells, neurons were visualized using a phase-contrast microscope (TE2000S; Nikon, Tokyo, Japan) with a $60 \times$ objective and $10 \times$ ocular lens. Voltage-clamp recordings were made using an Axoclamp 2A and pClamp 8 software. Electrical measurements used nystatin-perforated patch recordings at room temperature (Horn and Marty, 1988). Recording electrodes were filled with a solution composed of the following (in $\mathrm{mm}$ ): $50 \mathrm{KCl}, 100 \mathrm{~K}$ gluconate, and 10 HEPES; the $\mathrm{pH}$ of this solution was adjusted to 7.2 with Tris-OH. The final concentration of nystatin was $450 \mu \mathrm{g} / \mathrm{ml}$. Neurons dispersed in this manner have intact presynaptic boutons as indicated by the presence of spontaneous synaptic events: IPSCs and EPSCs (Jin et al., 2004). Neurons were voltage clamped to $-60 \mathrm{mV}$, and currents were sampled every $20 \mu$ sec and saved to computer. Data were analyzed off-line using pClamp 8 software and Mini Analysis Program (Synaptosoft, Decatur, GA).

Drugs. Agonist concentrations were chosen at $2 \times$ their $\mathrm{ED}_{50}$ value and antagonists were $10 \times \mathrm{ED}_{50}$. I, II, and III designated the respective mGluR group subtypes in association with the following agonist and antagonist drugs: NBQX, AP-5, L-(+)-2-amino-4-phosphonobutyric acid (L-AP-4; group III mGluR agonist), bicuculline methiodide $\left[\mathrm{GABA}_{\mathrm{A}}\right.$ receptor $\left(\mathrm{GABA}_{\mathrm{A}} \mathrm{R}\right)$ antagonist], $\left(2 S, 2^{\prime} R, 3^{\prime} R\right)-2-\left(2^{\prime}, 3^{\prime}\right.$-dicarboxycyclopropyl glycine (DCG4; group II mGluR agonist), ( $S$ )-3,5-dihydroxyphenyglycine (DHPG; group I mGluR agonist), $\left(2 S^{\prime}\right)$-2-amino-2-[(1S,2S')-2carboxycycloprop-1-yl]-3-(xanth-9-yl) propanoic acid (LY 341495; group II mGluR antagonist), (S)-(+)-a-amino-4-carboxy-2-methylbenzene acetic acid (LY 367385; mGluR I antagonist), and $(R, S)$ a-methylserine-O-phosphate (MSOP; group III mGluR antagonist). L-AP-4 and LY 341495 were dissolved in $0.1 \mathrm{~N} \mathrm{NaOH}$ at $10 \mathrm{~mm}$ before they were diluted with external solution. In a limited subset of experi- 
ments, isolated neurons were tested additionally for their sensitivity to activation of Glu release [spontaneous EPSCs (sEPSCs)] by two presynaptic receptors specific for unmyelinated and myelinated primary afferents, respectively: vanilloid receptor 1 (VR1) and $\mathrm{P}_{2} \mathrm{X}_{3}$ (Jin et al., 2004). The VR1 agonist capsaicin (CAP; $100 \mathrm{nM}$ ) was dissolved in ethanol at $1 \mathrm{~mm}$ before it was diluted with external solution. $\mathrm{P}_{2} \mathrm{X}_{3}$ receptors were activated by $\alpha \beta$ methylene ATP ( $\alpha \beta$-m-ATP; $10 \mu \mathrm{M})$. All of the drugs were obtained from Tocris Cookson (Ballwin, MO) except for $\alpha \beta$-m-ATP, which came from Sigma-RBI (Natick, MA). In isolated neurons, all of the drugs were applied via a rapid application Y-tube system that provided complete solution changes surrounding the recorded neurons within $20 \mathrm{msec}$ (Murase et al., 1989). In slices, drugs were applied by bath perfusion (bath volume, $0.7 \mathrm{ml}$; flow rate, $2 \mathrm{ml} /$ $\mathrm{min}$ ) providing for a bath turnover every $1 \mathrm{~min}$.

All data are presented as means \pm SEM. Statistical comparisons between two groups were made using unpaired Student's $t$ test. To evaluate differences for more than three groups, parameter values were compared by a repeatedmeasures ANOVA or one-way ANOVA (as appropriate) for the effects of treatments or groups. Post hoc comparisons of means used Bonferroni's correction for multiple comparisons where appropriate (Statview 4.57, Abacus Concepts, Cary, NC). $p$ values of $<0.05$ indicated significant differences.

\section{Results}

Presynaptic mGluRs modulate GABA terminals on second-order NTS neurons during afferent activation Glu acting at presynaptic mGluRs commonly depresses Glu or GABA release in cortical neurons (Schoepp, 2001; Schoepp and Conn, 2002). To test whether GABA release onto second-order medial NTS neurons (Fig. 1, inset) was altered by Glu released during ST activation, we recorded from neurons in brainstem slice preparations. We selected for study neurons with low-jitter ST-evoked EPSCs and then blocked those postsynaptic actions of Glu released by ST stimuli using ionotropic GluR blockade [data not shown but identical to published results (Doyle and Andresen, 2001; Bailey et al., 2002; Doyle et al., 2002)]. Ionotropic GluR blockade also eliminated ST-evoked polysynaptic IPSCs, as reported previously (Andresen and Yang, 1995). In the presence of ionotropic GluR blockade, all of the recorded second-order neurons $(n=25)$ exhibited spontaneous IPSCs (sIPSCs).

ST stimulation promptly decreased the rate of sIPSCs in some neurons (6 of 25) (Fig. 1, left), and we will refer to neurons in which the rate of sIPSCs is inhibited by Glu exposure as "decrease type.” In a second group of second-order NTS neurons (10 of 25), ST stimulation evoked a rapid increase in sIPSCs (Fig. 1, right), and we will refer to neurons in which the rate of sIPSCs is facilitated as "increase type." The remainder of second-order NTS neurons composed a third group of second-order NTS neurons, in which sIPSCs were not altered by ST stimulation ( 9 of 25; data not shown), and these unresponsive neurons were not studied
T3
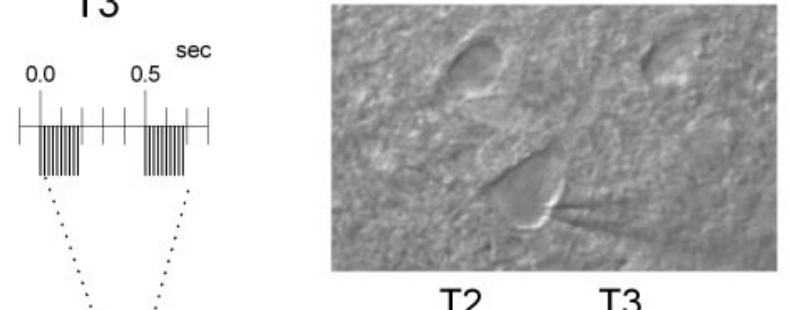

$\mathrm{T} 2$

T3

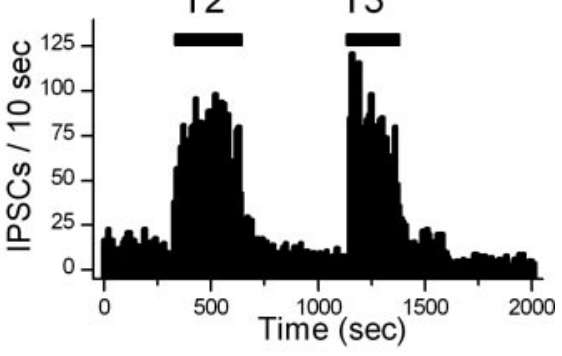

Control

T2
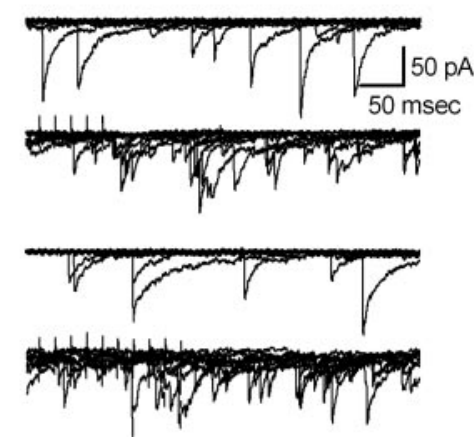

T3

Figure 1. IPSC regulation by mGluR on second-order medial NTS neurons in brainstem slices. After identifying second-order NTS neurons by their minimal ( $<150 \mu$ sec) synaptic jitter to ST activation (data not shown; see Materials and Methods), ST-

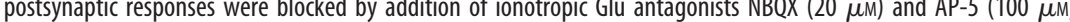
of the cell (top right micrograph). After ionotropic GluR blockade, large-amplitude, long-duration spontaneous GABAergic IPSCS ins (i.e., $50 \mathrm{~Hz})$, delivered as short bursts that were repeated every 0.5 sec as depicted at the top left; horizonta 列

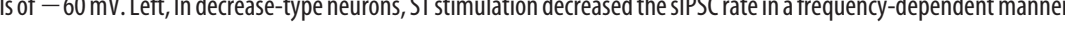
bottom, 10 consecutive original traces superimposed for each condition. Bin plots indicate that SIPSC rates increase more rapidly for T3 than T2. For clarity, stimulus artifacts have been truncated in some of the original traces.

further. The rapid actions of ST activation were clearest in increase-type neurons, because of the severalfold changes in sIPSC rate common in these neurons. Changes in IPSC frequency were graded with number of shocks per burst (T1, T2, and T3) and were well sustained over several minutes (Fig. 1, left). With the highest train frequencies (T3), increase-type responses clearly showed large, early transient increases in sIPSCs, even within the first 10 sec counting bin, and then settled to a lower sustained rate (Fig. 2B). On halting ST stimulation, sIPSC rates dropped promptly to near control levels (Fig. 1). The rapid onset and recovery allowed responses to be measured repeatedly within single neurons.

The pattern of modulation of sIPSCs in slices indicated a selectively presynaptic mechanism to modulate the frequency of GABA release. Mean amplitudes of the sIPSCs were not changed by ST stimulation in any of the neurons $(p>0.05)$, and holding currents were not altered (decrease type: $p=0.39, n=6$, $-43.3 \pm 42.7 \mathrm{pA} v \mathrm{vs}-40 \pm 39.3 \mathrm{pA}$, control and $\mathrm{T} 3$ stimulation, 


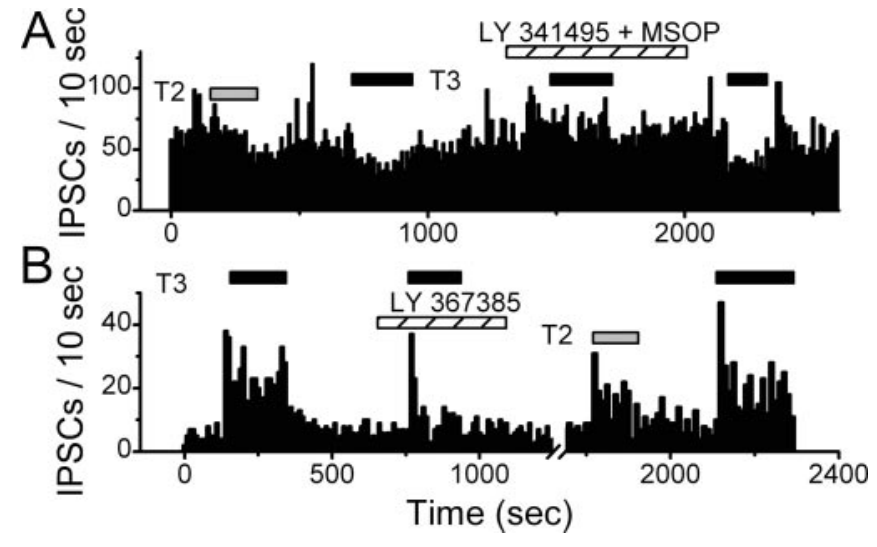

Figure 2. Subtype-specific mGluR receptor antagonists block the effects of ST activation on SIPSCs. In brainstem slices (see Fig. 1 for conditions), ST stimulation modulated the frequency of sIPSCs in subtype-representative second-order NTS neurons. A, Decrease-type neuron. T2 and T3 (horizontal bars) depressed SIPSCs. Modest concentrations of combined group II (LY 341495; $20 \mathrm{nM}$ ) and group III (MSOP; $200 \mu \mathrm{M}$ ) mGluRantagonists reversibly blocked the highest intensity (T3) of ST-induced depression of GABA release, and this block was rapidly reversed by return to control perfusate. $B$, Increase-type neuron. High-frequency ST volley (T3) abruptly increased SIPSC frequency that rapidly returned to control after cessation of ST stimulation. Group I mGluR antagonism (LY 367385; $160 \mu \mathrm{m}$ ) strongly attenuated T3 ST stimulation-induced increases in GABA release. Blockade reversed rapidly with control perfusion so that $\mathrm{T} 2$ and $\mathrm{T} 3$ volleys produced frequency-graded increases in sIPSC frequency.

respectively; increase type: $p=0.09, n=10,-42.7 \pm 25.2 \mathrm{pA}$ vs $-44.5 \pm 25.2 \mathrm{pA}$, control and T3 stimulation, respectively).

To identify the receptor responsible for ST modulation of GABA release, we tested whether group-specific mGluR antagonists blocked these effects (Fig. 2). In decrease-type NTS secondorder neurons, ST-evoked reductions in SIPSC rates were effectively prevented by a combination of group II and group III antagonists, LY $341495(20 \mathrm{nM})$ and MSOP $(200 \mu \mathrm{M})$, respectively (Fig. 2A). In the increase-type NTS second-order neurons (Fig. $2 B$ ), ST-evoked increases in sIPSC rates were effectively attenuated by the group I mGluR antagonist LY $367385(60 \mu \mathrm{M})$ alone. The early burst in sIPSCs in increase-type responses was not always blocked by group I mGluR antagonist (Fig. $2 B$, middle epoch). In no case was a decrease-type response converted to an increase-type response in the presence of antagonist. ST modulation of GABA release was restored within $<5$ min of returning to antagonist-free perfusate (Fig. 2).

Overall, ST stimulation modulated sIPSC rate in a graded manner with ST activation (Fig. 3). In decrease-type neurons, the medium strength burst protocol (T2) produced an $\sim 40 \%$ reduction in GABA release, and these responses were reversed completely within $5 \mathrm{~min}$ (Fig. 3A). Antagonists were compared with T3 stimuli, the strongest ST trains. Remarkably, the increase-type responses in second-order NTS neurons appeared to be nearly maximal at five shocks every $0.5 \mathrm{sec}$ (T2) (Fig. 3B), so that T3 was not significantly different from T2. In increase-type neurons, even two ST shocks every $0.5 \mathrm{sec}$ significantly increased the GABA release rate by nearly $50 \%$ (Fig. $3 B, \mathrm{~T} 1$ ). Antagonists alone in any of these neurons did not alter release rates (Fig. $3 A, B$ ). Combined application of group II and group III mGluR antagonist to decrease-type neurons completely blocked the response to T3 ST stimulation. Group I antagonist effectively attenuated the average increase to T3 ST stimulation. Close inspection of the records suggests that an initial spike in IPSCs occurred in many neurons at the earliest phase of the response (Fig. $2 B$ ).
Mechanically isolated NTS neurons retain functioning Glu and GABA synaptic boutons

Within NTS, second-order neurons are intermixed with interneurons and projection neurons (Loewy, 1990). The complexity of testing multiple selective agonists and antagonists to identify mGluR subtypes was difficult to accomplish in slices, so we turned to dissociated NTS neurons. To isolate individual NTS neurons, we gently applied a vibrating stylus to the medial subnucleus of NTS in horizontally cut brainstem slices (Jin et al., 2004). Without enzyme treatment or trituration, the resulting isolated NTS neurons (Fig. 4A) retained somal shapes and proximal processes, and many closely resembled identified secondorder NTS neurons viewed in slices (Doyle and Andresen, 2001). Recordings from all of the isolated neurons revealed synaptic events from retained perisomatic synaptic terminals (Fig. 4) (Jin et al., 2004). Spontaneous release of neurotransmitter produced synaptic currents with two kinetically and pharmacologically distinct classes of events (Fig. 4). Large-amplitude synaptic currents decayed with a prolonged time course and were selectively blocked by the $\mathrm{GABA}_{\mathrm{A}} \mathrm{R}$ antagonist bicuculline, leaving smallamplitude events. The non-NMDAR antagonist NBQX reversibly blocked all of the small-amplitude events (Fig. 4). Combined NBQX and bicuculline together eliminated all of the spontaneous postsynaptic currents in these NTS neurons. GABA ${ }_{\mathrm{A}}$ sIPSCs were isolated for study by recording in the continuous presence of ionotropic GluR blockade (NBQX, $20 \mu \mathrm{M}$; AP-5, $100 \mu \mathrm{M}$ ) in dissociated neurons.

\section{Subtypes of mGluRs oppositely modulate GABA release on different NTS neurons}

In every isolated NTS neuron, exogenous Glu (1 mM) altered the sIPSC rate. In individual neurons, however, Glu either increased or decreased the sIPSC rate (Fig. 5). Glu most commonly reduced the frequency of sIPSCs in dispersed NTS neurons (36 of 42 neurons) (Fig. 5A). In contrast, in the remainder of the neurons (6 of 42), Glu substantially increased the rate of GABA release (Fig. $5 B$ ). Note that in both types of responses, the sIPSC frequency changed quite rapidly and was sustained during Glu application. On returning the Y-tube delivery to control perfusate, release rates rapidly recovered to control levels. Thus, mGluR activation and deactivation generally altered frequency reversibly within the first $10 \mathrm{sec}$ count bin (Fig. 5A,B).

On average, in dissociated NTS neurons (Fig. 6), decreasetype neurons were more common than increase-type neurons, and in both types, mGluR effects were selectively presynaptic. Note that, unlike in slice studies, no NTS neurons were found with unresponsive IPSCs. On average, for a $60 \mathrm{sec}$ Glu protocol in decrease-type neurons $(n=22)$, Glu depressed the frequency of sIPSCs by nearly $70 \%$ from control levels (from $0.66 \pm 0.14$ to $0.20 \pm 0.04 \mathrm{~Hz} ; p=0.0001)$ without altering the mean amplitudes $(19.3 \pm 4.0$ vs $17.1 \pm 3.6 \mathrm{pA}$, control and Glu, respectively; $p=0.44)$. In increase-type neurons $(n=6)$, for the $60 \mathrm{sec}$ protocol, Glu triggered a nearly fourfold increase in the sIPSC event rate $(0.41 \pm 0.17$ vs $1.63 \pm 0.66 \mathrm{~Hz} ; p=0.02)$, again without changing sIPSC amplitudes (17.2 \pm 7.0 to $19.0 \pm 7.8 \mathrm{pA} ; p=$ $0.19)$. In the presence of ionotropic blockers, Glu did not alter holding currents in either decrease-type neurons $(-34.4 \pm 4.1 \mathrm{vs}$ $-41.1 \pm 6.2 \mathrm{pA}$, control and Glu, respectively; $n=20$ ) or increase-type neurons $(-65.8 \pm 21.2$ vs $-54.3 \pm 27.2 \mathrm{pA}$, control and Glu, respectively; $n=4)$. Such results suggest that Glu acts by selective presynaptic actions without affecting postsynaptic $\mathrm{GABA}_{\mathrm{A}}$ or mGluR targets. 


\section{Group II-III mGluRs decrease GABA release}

With our isolated NTS neurons, multiple agonist-antagonist tests in single neurons were possible with the Y-tube rapid perfusion system (Jin et al., 2004). Group II (DCG4) and group III (L-AP-4) mGluR agonists mimicked the effects of exogenous Glu application within individual decrease-type neurons (Fig. 7). Separately applied, DCG4 $(10 \mu \mathrm{M})$ and L-AP-4 (10 $\mu \mathrm{M})$ each reversibly decreased sIPSC frequency (Fig. 7). In such decrease-type neurons, the mGluR group I-selective agonist DHPG $(10 \mu \mathrm{M})$ did not change the sIPSC frequency (Fig. 7). In decrease-type neurons, combining group II (LY 341495; $20 \mu \mathrm{M}$ ) and group III (MSOP; $200 \mu \mathrm{M}$ )selective antagonists effectively blocked the actions of exogenous Glu on sIPSC rate (Fig. 7). These antagonists individually failed to block Glu-induced reductions in IPSCs (Fig. 7, right). Together, the agonist and antagonist test series suggest that the decrease-type NTS neurons possess a mixture of both group II and group III mGluRs on GABA terminals that depress release after activation.

\section{Group I mGluRs facilitate GABA release}

In increase-type neurons, Glu increased sIPSC rates more than threefold without altering the IPSC amplitudes (Fig. 8A,B). DHPG closely mimicked the Glu-evoked increase in sIPSC frequency. All of the mGluR agonists appear to act selectively at presynaptic sites in isolated medial NTS neurons. As with Glu itself, mGluR agonists did not alter holding current regardless of neuron response type $(p>0.05)$. In the presence of ionotropic blockers, DHPG did not alter holding currents in either decrease-type neurons $(-26.8 \pm$ 9.4 vs $-29.2 \pm 9.4 \mathrm{pA}$, control and DHPG, respectively; $n=4)$ or increase-type neurons $(-54.8 \pm 24.8$ vs $-54.5 \pm 24.9 \mathrm{pA}$, control and DHPG, respectively; $n=4)$. Likewise, DCG4 did not alter holding currents in either decrease-type neurons $(-23.8 \pm$ 6.4 vs $-23.6 \pm 6.0 \mathrm{pA}$, control and DCG4, respectively; $n=3$ ) or increase-type neurons $(-29.0 \pm 1.0 \mathrm{vs}-27.0 \pm 4.0 \mathrm{pA}$, control and DCG4, respectively; $n=2$ ). Holding currents were not altered by L-AP-4 in either decrease-type neurons $(-32.4 \pm 6.5$ vs $-32.4 \pm 6.1 \mathrm{pA}$, control and L-AP-4, respectively; $n=5$ ) or increase-type neurons $(-74.0 \pm 45.1$ vs $-76.9 \pm 44.4 \mathrm{pA}$, control and L-AP-4, respectively; $n=3$ ). The relatively limited numbers of these neurons prevented our testing all of the compounds on every neuron. The constant holding current, together with agonist actions on frequency but not amplitude of sIPSCs during mGluR activation, are consistent with selective presynaptic expression of mGluRs at these GABA synapses.

\section{Increase- and decrease-type neurons receive either myelinated or unmyelinated afferents}

Two classes of second-order neurons may be distinguished by their differential expression of VR1 or $\mathrm{P}_{2} \mathrm{X}_{3}$ receptors on their ST afferent terminals (Jin et al., 2004). This group of isolated NTS neurons was tested for afferent subtype at the end of the mGluR protocols. In these neurons, sIPSCs were blocked with bicucul- line, and sEPSCs were recorded after ionotropic antagonists were removed from the perfusate. Under these conditions, neurons $(n=$ 14) were then challenged with either CAP or $\alpha \beta$-m-ATP, or both, sequentially (Jin et al., 2004). In agonist tests of EPSC sensitivity in decrease-type neurons $(n=12)$, four had CAP-sensitive Glu terminals, six had $\alpha \beta$-m-ATP-sensitive Glu terminals, and two were negative for both afferent agonists. In the agonist responsive neurons, activating VR1 was not effective in $\mathrm{P}_{2} \mathrm{X}_{3}$-sensitive neurons and vice versa. Only two increase-type neurons were recorded for this series; one was responsive to $\alpha \beta$-m-ATP but not CAP, and the other did not respond to CAP. As observed previously (Jin et al., 2004), individual neurons responding to either CAP or $\alpha \beta$-m-ATP would be considered second-order NTS neurons.

\section{Discussion}

The rich diversity of the mGluR family provides the potential for multiple distinct modes of CNS regulation through processes ranging from modulation of transmitter release to alteration of discharge properties. Although multiple different subtypes of mGluRs may be expressed within single brain regions, identifying their contributions within subnuclei is complicated by heterogeneous expression patterns and functional interactions among local pools of neurons. Varied mGluR actions reported in early investigations of NTS hinted at diversity (Feldman and Felder, 1991; Glaum et al., 1993). Using dissociated and slice neurons, our studies provide direct evidence for a differential distribution of mGluRs among GABA terminals in NTS. GABA terminals on second-order NTS neurons express a distinct pattern of mGluR expression that mediates opposite regulation of GABA release by cranial afferent Glu. 

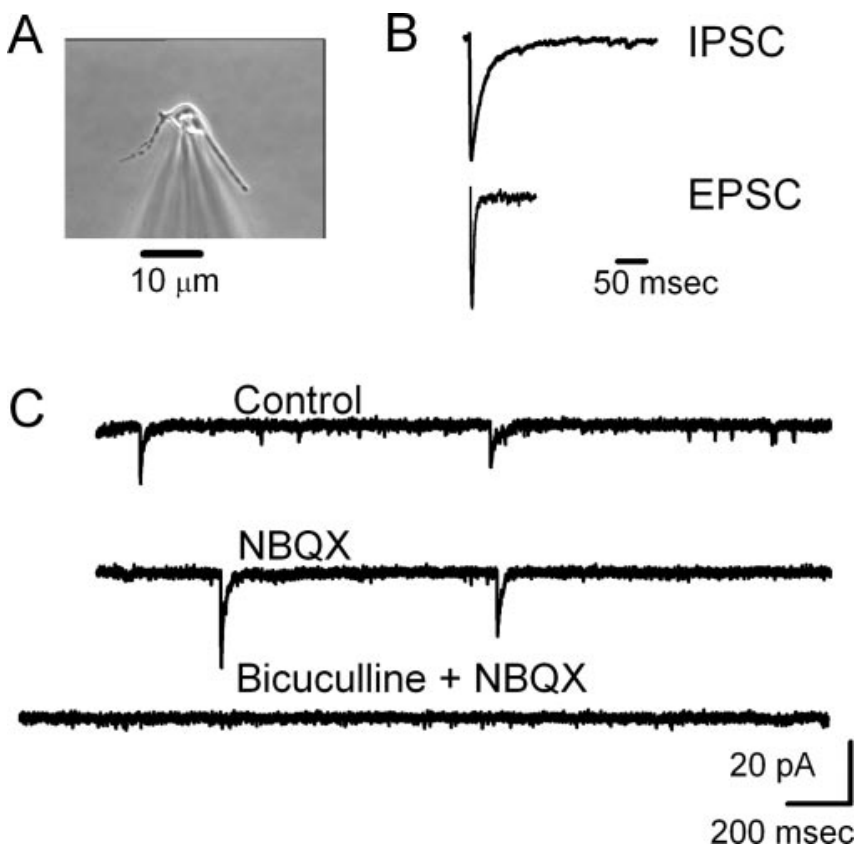

Figure 4. Isolated NTS neurons were dispersed from the medial region of NTS for recording of spontaneous synaptic currents. A, Spindle-shaped neurons were selected for recording because they closely resembled second-order NTS neurons found in slices. B, All of the neurons had spontaneous synaptic currents in control conditions (top single-sweep trace). IPSCs were isolated for study by blocking EPSCs with NBQX (20 $\mu \mathrm{m}$; middle trace). Note that under our recording conditions (internal $\mathrm{Cl}^{-} 50 \mathrm{~mm}$ ) and a holding potential of $-60 \mathrm{mV}$, IPSCS were inward. Combined antagonist block (lowest trace) with bicuculline $(100 \mu \mathrm{M})$ and NBQX eliminated all of the synaptic events. C, Clear kinetic differences also distinguished IPSCs from EPSCs in this same neuron. Expanded mean traces illustrate the prolonged time course of IPSCs compared with the rapid time course of EPSCS (amplitudes normalized to 100\%). The IPSC and EPSC traces are averages of 69 and 80 synaptic currents, respectively. All of the traces and the photo are from the same NTS neuron.

\section{Heterogeneous, group-specific mGluR modulation of GABA transmission}

The second-order neurons within the medial portions of caudal NTS are readily identified by electrophysiological criteria and receive GABAergic as well as afferent Glu synapses (Doyle and Andresen, 2001). By blocking ionotropic GluR, the present studies revealed distinct changes in GABA release onto these NTS neurons during afferent ST stimulation and the presence of three mGluR response types in slices: increase, decrease, and unresponsive. NTS neurons mechanically dissociated from the same subnucleus retained GABAergic terminals that responded similarly to Glu and mGluR agents. No dissociated neurons failed to respond to Glu with changes in sIPSC rate.

Using mGluR-selective agents, the increase and decrease types of GABA regulation were associated with group I mGluRs and combined group II-III mGluRs, respectively. In isolated neurons, group-selective mGluR agonists mimicked Glu actions, and group-specific antagonists likewise blocked the actions of both Glu and group-specific mGluR agonists. Selective group I agonist facilitated GABA release, and selective group II or group III agonists depressed sIPSCs. Accordingly, group-specific antagonists eliminated agonist responses, and in no case was an opposite signed response uncovered (i.e., increase-type responses converted to decrease type or vice versa).

The degree of concordance of the results between slice and dissociated cell was remarkable. ST stimulation either increased or decreased sIPSCs in slice studies of second-order neurons, although the proportion of each response type was dissimilar.

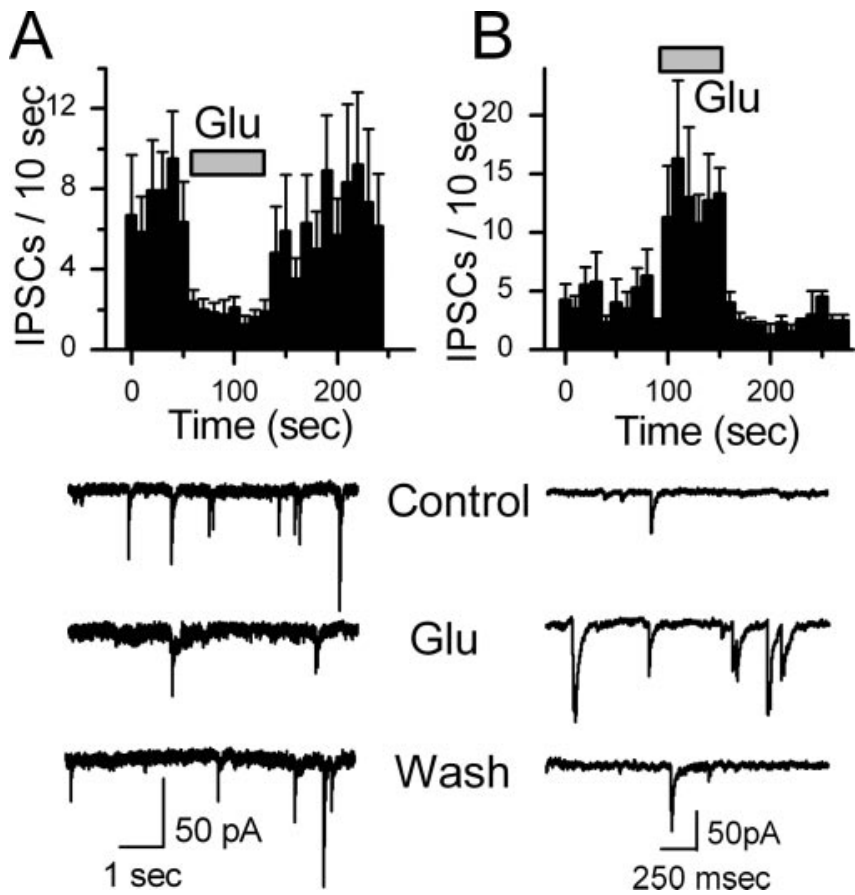

Figure 5. Exogenous Glu applied to dispersed NTS neurons either depressed or increased the spontaneous release of GABA (sIPSCS) in different NTS neurons. In all of the experiments, ionotropic Glu responses were blocked with NBQX $(20 \mu \mathrm{M})$ and AP-5 (100 $\mu \mathrm{M})$. IPSC detection counts in all of the cases were collected in 10 sec bins, and points are means \pm SEM. $A$, Decrease-type NTS neurons $(n=10)$. Glu application (1 mM; horizontal bar) rapidly decreased SIPSC frequency in decrease-type neurons, and this effect was rapidly reversed after switching back to control solution. Bottom, Representative samples of sIPSCS recorded from a single neuron during control, Glu, and wash with control solution. $B$, Increase-type NTS neurons ( $n=$ 5). Glu increased the sIPSC frequency averaged at each time bin. Bottom, Representative samples from a single neuron during control, Glu, and wash with control solution.

Some second-order NTS neurons had sIPSC rates that were unresponsive to ST stimulation. Given the consistency of isolated neurons in their mGluR responses, the unresponsive slice neurons may reflect damage to ST connections to GABA terminals in preparing slices. In dissociated cells, conducted responses are not necessary, because GABA events arise from isolated terminals and $m G l u R$ responses were evoked by exogenous agents. In slices, variability in rates of GABA release may reflect activity from local circuits that may obscure the smaller mGluR group II-III responses in slices more than in isolated cells. The slice studies focused on a select subpopulation of NTS neurons: second-order neurons. The dissociated work may include a more diverse population (e.g., higher-order neurons). Consistent with that view, we recorded two dissociated neurons with EPSCs that were not responsive to either CAP or $\alpha \beta$-m-ATP (nonafferent profile) but nonetheless showed a decrease-type mGluR sIPSC response. We speculate that this nonafferent agonist profile reflects a higher than second-order NTS neuron that is not included in the slice work.

Our results are consistent with the conclusion that no mixed populations of group I with group II-III mGluR occur on NTS GABA terminals. If mGluR intermixing had been the case, subtype-selective antagonism would have produced oppositely coupled mGluR responses, yet such reversed responses were never observed. Thus, our overall results support a differential distribution of distinct mGluRs on GABA terminals that populate different NTS neurons. 


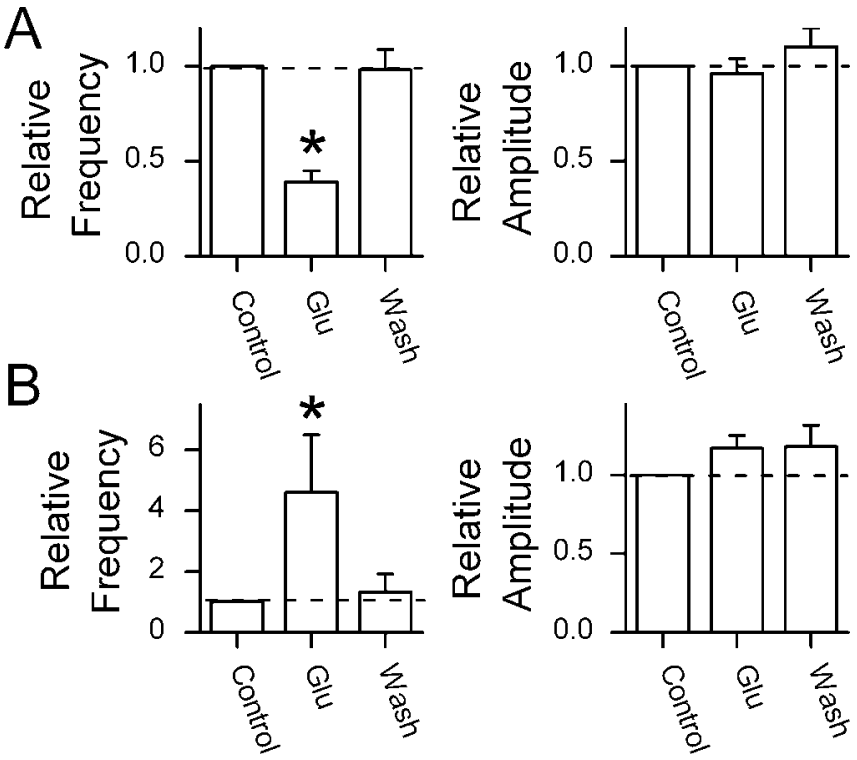

Figure 6. Glutamate acts via presynaptic mechanisms at both increase- and decrease-type NTS neurons. Broken line indicates control sIPSC levels normalized to 1.0. *Significant difference from control $(p<0.05)$. A, The sIPSC rate in decrease-type NTS neurons $(n=22)$ was reversibly reduced by application of exogenous $\mathrm{Glu}(1 \mathrm{mM})$. Amplitudes of sIPSCs were unaltered in all three of the conditions. $B$, In increase-type neurons $(n=6)$, Glu increased the mean frequency of spontaneous GABAergic IPSCs by nearly fivefold. Glu did not alter sIPSC mean amplitudes in increase-type neurons.

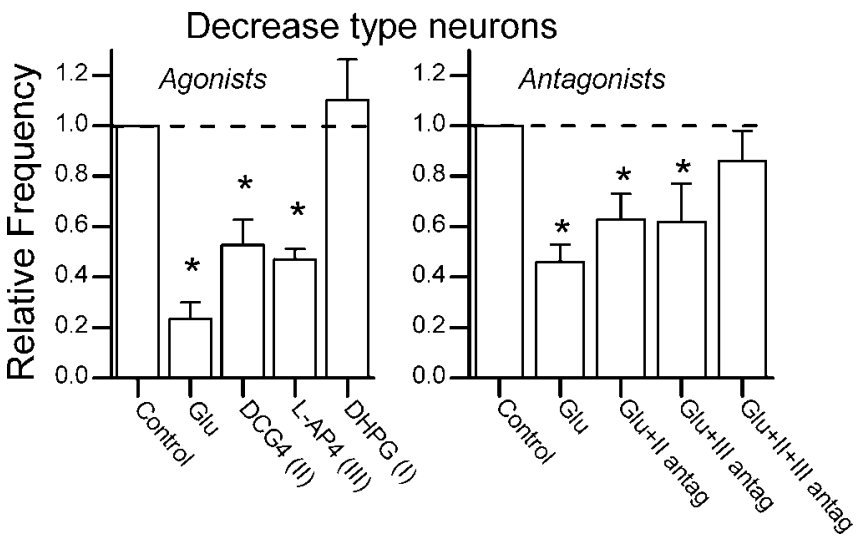

Figure 7. Selective group II and group III mGluR drug actions on decrease-type NTS neurons. Bins of sIPSCs were counted over intervals of $10 \mathrm{sec}$. Left, Glu (1 mM) substantially depressed SIPSC rates in decrease-type neurons $(n=12 ; p<0.0001)$. The group II mGluR-selective agonist DCG4 $(10 \mu \mathrm{m} ; n=6)$ or the group III mGluR-selective agonist L-AP-4 $(10 \mu \mathrm{m} ; n=8)$ mimicked the actions of Glu in these neurons $(p<0.02)$. Not all of the neurons were tested for all of the agents. Four of these neurons responded similarly to Glu as well as DCG4 and L-AP-4 (within-neuron tests). The group I mGluR-selective agonist DHPG (10 $\mu \mathrm{m} ; n=6)$ failed to alter sIPSCs ( $p=0.58)$. Five of these DHPG-insensitive neurons were sensitive to L-AP-4, and three of these DHPG-insensitive neurons were sensitive to L-AP-4 and DCG4. Right, In a different set of tests of neurons $(n=12)$, Glu (1 mM) depressed sIPSC rate by more than half $(p<0.001)$. Combined antagonists for group II (LY 341495; $10 \mathrm{nM}$ ) and group III (MSOP; $200 \mu \mathrm{M}$, ) blocked the Glu effects $(n=7 ; p>0.3)$. In five of the neurons tested with II-III combined, either group II antagonist $(n=4)$ or group III antagonist $(n=4)$ was additionally tested separately. These single subtype-selective antagonists failed to eliminate Glu responses $(p<0.05)$. Three of the seven neurons subjected to II-III combined drugs were tested separately for both antagonists as well. The data suggest that both group II and group III mGluR receptors are present on GABA terminals of decrease-type neurons, but these neurons are devoid of group I mGluR receptors.

\section{ST cranial visceral afferent subtypes}

ST afferents deliver a major glutamatergic drive to NTS, and second-order neurons can be subdivided by afferent subtype using pharmacological criteria [myelinated and unmyelinated, as-

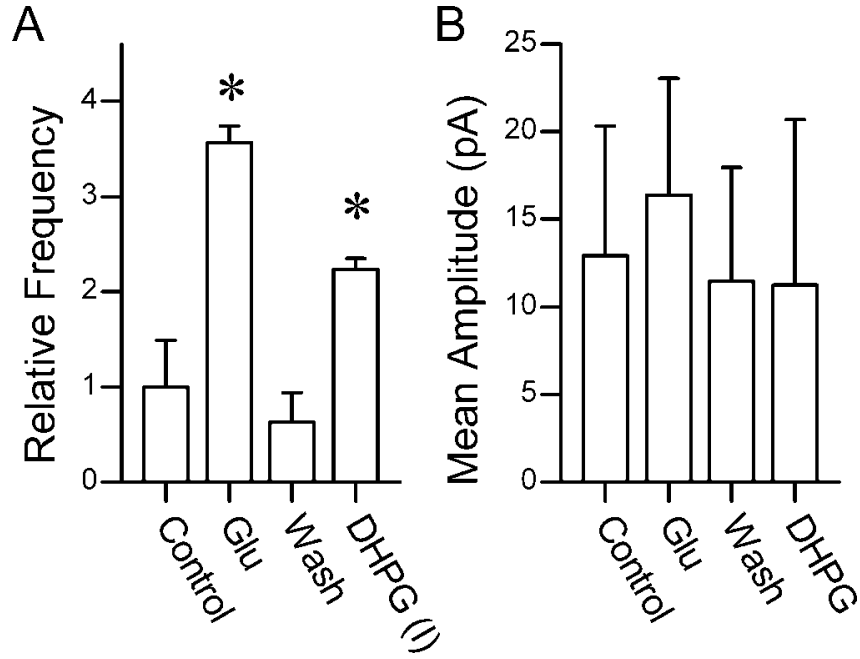

Figure 8. In increase-type NTS neurons $(n=3)$, group I mGluR agonist mimicked Glu on sIPSCs. Activation of group I mGluRs with Glu (1 mM) or with DHPG (10 $\mu \mathrm{M})$ reversibly increased the frequency of sIPSCS $(A)$. Neither Glu nor DHPG altered sIPSC amplitudes $(B)$, supporting a selective presynaptic group I mGluR action on GABA terminals. All of the neurons completed the same drug protocol: Glu, wash, and DHPG. *Significant difference from control ( $p<0.05)$.

sociated with $\mathrm{P}_{2} \mathrm{X}_{3}$ and VR1, respectively (Bailey et al., 2002; Doyle et al., 2002; Jin et al., 2004)]. Here, we found no evidence to support a distinct association between a particular type of afferent innervation and the type of mGluR-GABA terminals on individual NTS neurons. Decrease-type mGluR-GABA regulation was evident in neurons with either CAP-sensitive or $\alpha \beta$-m-ATPsensitive Glu terminals (i.e., C-type or A-type afferent terminals, respectively). This finding seems sufficient to conclude that there is no direct correlation between ST afferent subtype and the presence of decrease-type regulation of GABA terminals. Such results suggest that the NTS neurons displaying increase-decrease types of GABA regulation are not confined to a single afferent pathway subtype.

\section{Rapid, sensitive presynaptic mGluR modulation of GABA release}

An impressive feature of mGluR responses at GABA terminals was their speed of onset and recovery. Rapidly coupled mGluR characteristics were evident across both slice and dissociated cell preparations, despite substantial differences in drug delivery and recording conditions (e.g., temperature). Heterosynaptic Glu spillover (Schoepp, 2001) to activate mGluRs on GABA terminals in NTS was most obvious in increase-type response pattern because sIPSC rates jumped severalfold. GABA release increased as early as $60-70 \mathrm{msec}$ after the first ST shocks in successive afferent bursts (Fig. 1, T3, right). The temporal resolution in detecting mGluR responses was limited by spontaneous GABA release rates (a few events per second) and thus the actual response rate could be underestimated. The sIPSC rate in isolated neurons increased or decreased within a few seconds of exogenous Glu delivery and recovered equally quickly. Quantitatively, afferent fiber activation significantly changed sIPSC rates at minimal ST frequencies (pairs of shocks per burst; T1) that are similar to established afferent discharge frequencies in the intact state [e.g., baroreceptors (Kunze and Andresen, 1991; Pamidimukkala and Hay, 2004)]. Although the receptor coupling mechanisms for NTS mGluR-GABA responses are unknown, G-protein coupling to ion channels can be quite rapid (Jin and Akaike, 1998).

Activation of mGluRs in many central neurons evokes sub- 
stantial postsynaptic currents, including reports in NTS (Glaum et al., 1993). In general, group I mGluRs modulate postsynaptic ion channels to decrease excitability, whereas group II and group III mGluRs commonly act presynaptically to depress transmitter release (Cartmell and Schoepp, 2000; Schoepp and Conn, 2002). Heteroceptor mGluRs most often depress GABA release [e.g., group I mGluRs in hippocampus (Conn and Pin, 1997)]; however, a recent report (Fiacco and McCarthy, 2004) showed that group I mGluR activation increased Glu release in CA1 pyramidal neurons. In discriminating mGluR subtypes, selective agents and control of their concentration are critical factors, and selective agonists are particularly useful (Cartmell and Schoepp, 2000). For example, DHPG effectively selects for group I mGluRs over group II or III by a $>100$-fold difference in $\mathrm{EC}_{50}$ values. Many antagonists have overlapping activities [e.g., $\alpha$-methyl-4carboxyphenylglycine (MCPG); group I and group II] or discriminate poorly ( $<10$-fold) among subtypes of mGluRs (e.g., MPPG). Thus, the selection of tools and multiple tests are necessary to discriminate subgroup participation.

Because mGluR expression is quite broad within NTS, other subregions and particular neuron types within NTS may have different sensitivities [e.g., afferent triggered EPSCs insensitive to group I interventions (Chen et al., 2002)]. Here, we found no evidence of postsynaptic actions of mGluRs in second-order NTS neurons. Our perforated-patch recordings should minimize disruptions of intracellular signaling. Widespread expression of mGluR mRNA and subunits within NTS may reflect multiple populations of neurons and important heterogeneity within the nucleus. Regional differences may account for the failure of focal stimulation with caged Glu to activate mGluR on GABA terminals (Davis et al., 2003) or the failure of the broad-spectrum mGluR (I-II) antagonist MCPG injected into commissural NTS to alter cardiovascular reflexes (Antunes and Machado, 2003). Thus, it may be particularly important to identify the neurons involved, given heterogeneity in mGluR expression.

The functional impact of distinct mGluR regulation of ST inputs to NTS second-order neurons is unknown. Some GABA terminals likely originate from local interneurons, whereas others may well arise from outside NTS (Jordan et al., 1988). Shortlatency, afferent-synched IPSCs (Mifflin and Felder, 1988; Andresen and Yang, 1995) appear to reflect local feedback circuits onto second-order NTS neurons. Precisely which circuits provide particular GABA terminals in NTS will dictate their ultimate contribution to pathway function. Interestingly, the discrete separation of group I from group II-III mGluRs on second-order NTS neurons may presage pathway specificity that we can presently only anticipate. Neither broad class of afferent (myelinatedunmyelinated) was uniquely associated with a particular type of mGluR-GABA regulation. Given the low frequencies of afferent activity required as well as the power of mGluR actions on GABA release, these heterosynaptic interactions may be important for dynamic transmission of afferent signals through the nucleus along subsequent projection pathways.

The brainstem contains the core pathways that provide homeostatic control for autonomic regulation (Andresen and Kunze, 1994; Pilowsky and Goodchild, 2002). Even at rest, many pathways are constantly engaged by primary visceral afferent activity (Andresen and Kunze, 1994). Cardiovascular control pathways are particularly prominent, and mGluRs participate in their regulation within NTS (Foley et al., 1999). Our results add powerful interactions between glutamatergic afferent drive and GABAergic feedback. Because broad-spectrum mGluR antagonists increase NTS activity during vagal and aortic baroreceptor afferent activation (Liu et al., 1998), understanding the interactions between glutamatergic and GABAergic transmission will depend on the unraveling of specific interrelations between distinct subsets of neurons to define their integrative properties.

\section{References}

Andresen MC, Kunze DL (1994) Nucleus tractus solitarius: gateway to neural circulatory control. Annu Rev Physiol 56:93-116.

Andresen MC, Yang M (1990) Non-NMDA receptors mediate sensory afferent synaptic transmission in medial nucleus tractus solitarius. Am J Physiol 259:H1307-H1311.

Andresen MC, Yang M (1995) Dynamics of sensory afferent synaptic transmission in aortic baroreceptor regions of nucleus tractus solitarius. J Neurophysiol 74:1518-1528.

Andresen MC, Doyle MW, Jin Y-H, Bailey TW (2001) Cellular mechanisms of baroreceptor integration at the nucleus tractus solitarius. Ann NY Acad Sci 940:132-141.

Antunes VR, Machado BH (2003) Antagonism of glutamatergic metabotropic receptors in the NTS of awake rats does not affect the gain of the baroreflex. Auton Neurosci 103:65-71.

Bailey TW, Jin Y-H, Doyle MW, Andresen MC (2002) Vanilloid-sensitive afferents activate neurons with prominent A-type potassium currents in nucleus tractus solitarius. J Neurosci 22:8230-8237.

Cartmell J, Schoepp DD (2000) Regulation of neurotransmitter release by metabotropic glutamate receptors. J Neurochem 75:889-907.

Chen CY, Ling Eh EH, Horowitz JM, Bonham AC (2002) Synaptic transmission in nucleus tractus solitarius is depressed by group II and III but not group I presynaptic metabotropic glutamate receptors in rats. J Physiol (Lond) 538:773-786.

Conn PJ, Pin JP (1997) Pharmacology and functions of metabotropic glutamate receptors. Annu Rev Pharmacol Toxicol 37:205-237.

Davis SF, Williams KW, Xu W, Glatzer NR, Smith BN (2003) Selective enhancement of synaptic inhibition by hypocretin (orexin) in rat vagal motor neurons: implications for autonomic regulation. J Neurosci 23:3844-3854.

Doyle MW, Andresen MC (2001) Reliability of monosynaptic transmission in brainstem neurons in vitro. J Neurophysiol 85:2213-2223.

Doyle MW, Bailey TW, Jin Y-H, Andresen MC (2002) Vanilloid receptors presynaptically modulate visceral afferent synaptic transmission in nucleus tractus solitarius. J Neurosci 22:8222-8229.

Doyle MW, Bailey TW, Jin Y-H, Appleyard SM, Low MJ, Andresen MC (2004) Strategies for cellular identification in nucleus tractus solitarius slices. J Neurosci Methods 37:37-48.

Feldman PD, Felder RB (1991) Effects of gamma-aminobutyric acid and glycine on synaptic excitability of neurones in the solitary tract nucleus. Neuropharmacology 30:225-236.

Fiacco TA, McCarthy KD (2004) Intracellular astrocyte calcium waves in situ increase the frequency of spontaneous AMPA receptor currents in CA1 pyramidal neurons. J Neurosci 24:722-732.

Foley CM, Vogl HW, Mueller PJ, Hay M, Hasser EM (1999) Cardiovascular response to group I metabotropic glutamate receptor activation in NTS. Am J Physiol 276:R1469-R1478.

Glaum SR, Sunter DC, Udvarhelyi PM, Watkins JC, Miller RJ (1993) The actions of phenylglycine derived metabotropic glutamate receptor antagonists on multiple $(1 S, 3 R)$-ACPD responses in the rat nucleus of the tractus solitarius. Neuropharmacology 32:1419-1425.

Hay M, McKenzie H, Lindsley K, Dietz N, Bradley SR, Conn PJ, Hasser EM (1999) Heterogeneity of metabotropic glutamate receptors in autonomic cell groups of the medulla oblongata of the rat. J Comp Neurol 403:486-501.

Headley PM, Grillner S (1990) Excitatory amino acids and synaptic transmission: the evidence for a physiological function. Trends Pharmacol 11:205-211.

Hoang CJ, Hay M (2001) Expression of metabotropic glutamate receptors in nodose ganglia and the nucleus of the solitary tract. Am J Physiol Heart Circ Physiol 281:H457-H462.

Horn R, Marty A (1988) Muscarinic activation of ionic currents measured by a new whole-cell recording method. J Gen Physiol 92:145-159.

Jin YH, Akaike N (1998) Tandospirone-induced $\mathrm{K}^{+}$current in acutely dissociated rat dorsal raphe neurones. Br J Pharmacol 124:897-904. 
Jin YH, Bailey TW, Li BY, Schild JH, Andresen MC (2004) Purinergic and vanilloid receptor activation releases glutamate from separate cranial afferent terminals. J Neurosci 24:4709-4717.

Jordan D, Mifflin SW, Spyer KM (1988) Hypothalamic inhibition of neurons in the nucleus tractus solitarius of the cat is GABA mediated. J Physiol (Lond) 399:389-404.

Kunze DL, Andresen MC (1991) Arterial baroreceptors: excitation and modulation. In: Reflex control of the circulation (Zucker IH, Gilmore JP, eds), pp 141-166. Boca Raton, FL: CRC.

Liu Z, Chen CY, Bonham AC (1998) Metabotropic glutamate receptors depress vagal and aortic baroreceptor signal transmission in the NTS. Am J Physiol 275:H1682-H1694.

Loewy AD (1990) Central autonomic pathways. In: Central regulation of autonomic functions (Loewy AD, Spyer KM, eds), pp 88-103. New York: Oxford UP.

Mendelowitz D, Yang M, Andresen MC, Kunze DL (1992) Localization and retention in vitro of fluorescently labeled aortic baroreceptor terminals on neurons from the nucleus tractus solitarius. Brain Res 581:339-343.

Mifflin SW, Felder RB (1988) An intracellular study of time-dependent cardiovascular afferent interactions in nucleus tractus solitarius. J Neurophysiol 59:1798-1813.

Murase K, Ryu PD, Randic M (1989) Excitatory and inhibitory amino acids and peptide-induced responses in acutely isolated rat spinal dorsal horn neurons. Neurosci Lett 103:56-63.

Pamidimukkala J, Hay M (2004) Frequency dependence of synaptic vesicle exocytosis in aortic baroreceptor neurons and the role of group III mGluRs. Brain Res 1006:215-224.

Pamidimukkala J, Hoang CJ, Hay M (2002) Expression of metabotropic glutamate receptor 8 in autonomic cell groups of the medulla oblongata of the rat. Brain Res 957:162-173.

Pilowsky PM, Goodchild AK (2002) Baroreceptor reflex pathways and neurotransmitters: 10 years on. J Hypertens 20:1675-1688.

Schoepp DD (2001) Unveiling the functions of presynaptic metabotropic glutamate receptors in the central nervous system. J Pharmacol Exp Ther 299:12-20.

Schoepp DD, Conn PJ (2002) Metabotropic glutamate receptors. Pharmacol Biochem Behav 74:255-256.

Sekizawa S, Joad JP, Bonham AC (2003) Substance P presynaptically depresses the transmission of sensory input to bronchopulmonary neurons in the guinea pig nucleus tractus solitarii. J Physiol (Lond) 552:547-559.

Standish A, Enquist LW, Escardo JA, Schwaber JS (1995) Central neuronal circuit innervating the rat heart defined by transneuronal transport of pseudorabies virus. J Neurosci 15:1998-2012.

Titz S, Keller BU (1997) Rapidly deactivating AMPA receptors determine excitatory synaptic transmission to interneurons in the nucleus tractus solitarius from rat. J Neurophysiol 78:82-91.

Wang J, Irnaten M, Neff RA, Venkatesan P, Evans C, Loewy AD, Mettenleiter TC, Mendelowitz D (2001) Synaptic and neurotransmitter activation of cardiac vagal neurons in the nucleus ambiguus. Ann NY Acad Sci 940: 237-246. 